\title{
EPOXIDAÇÃO DO ÓLEO DE MAMONA E DERIVADOS EMPREGANDO O SISTEMA CATALÍTICO $\mathrm{VO}(\mathbf{a c a c})_{2} / \mathrm{TBHP}^{\#}$
}

\author{
Marta Regina dos Santos Nunes* \\ Departamento de Física e Química, Universidade de Caxias do Sul, Rua Francisco Getúlio Vargas, 1130, 95070-560 \\ Caxias do Sul - RS, Brasil \\ Marcia Martinelli e Marcelo Monteiro Pedroso \\ Departamento de Química Inorgânica, Universidade Federal do Rio Grande do Sul, CP 15027, 91501-970 Porto Alegre - RS, \\ Brasil
}

Recebido em 25/1/08, aceito em 8/4/08, publicado na web em 29/4/08

\begin{abstract}
EPOXIDATION OF CASTOR OIL AND ITS DERIVATIVES USING VO(ACAC), /TBHP AS THE CATALYTIC SYSTEM. The alternative system $\mathrm{VO}(\mathrm{acac})_{2} / \mathrm{TBHP}$ was investigated for the epoxidation reaction of castor oil and its derivatives. Results of $88 \%$ of conversion, $73 \%$ of epoxidation and $82 \%$ of selectivity were obtained for the system containing $20 \%$ excess of TBHP and $1 \%$ of $\mathrm{VO}(\mathrm{acac})_{2}$ catalyst, during $3 \mathrm{~h}$ under toluene reflux. The product was characterized by GC/MS as methyl-cis-9, 10-epoxi, 12hydroxystearate and quantitative ${ }^{1} \mathrm{H}$ NMR was used to calculate the data above. Preliminary results indicate that the heterogeneous system $\mathrm{VO}(\mathrm{acac})_{2}$ grafted on $\mathrm{K} 10$ clay can also promote epoxidation of castor oil.
\end{abstract}

Keywords: epoxidation; castor oil; heterogeneous system.

\section{INTRODUÇÃO}

Óleos vegetais são importantes fontes renováveis de matériaprima para a indústria oleoquímica, pois além de oferecerem a possibilidade de um grande número de modificações estruturais, são materiais diversificados e de baixo custo. Estas características raramente podem ser encontradas em outras fontes industriais, fazendo com que os óleos vegetais sejam materiais de partida de grande importância sob os pontos de vista econômico, tecnológico e científico.

A diversidade das aplicações de óleos vegetais mostra a importância destes em gerar novos produtos e justifica o empenho no desenvolvimento de novas tecnologias, a fim de promover modificações em sua estrutura. Sendo assim, a investigação de novos processos industriais, bem como a modificação de processos clássicos de transformação química dos óleos vegetais, torna-se necessária para atender às necessidades econômicas atuais do país e às exigências de melhoria da qualidade de vida e preservação do meio ambiente. ${ }^{1}$

A aplicabilidade do óleo extraído da mamona (também chamado de óleo de rícino), da família Ricinus communis, é conhecida desde a era colonial, onde era usado para lubrificar os engenhos de cana-de-açúcar. Atualmente, o óleo de mamona tem sido extensivamente utilizado para fins industriais, devido às suas características físico-químicas, uma vez que suporta grandes e bruscas variações de temperatura. Além disso, é utilizado na fabricação de cosméticos, bem como na indústria de medicamentos. ${ }^{1}$

O principal constituinte do óleo de mamona é o triacilglicerol do ácido ricinoléico (aproximadamente 90\%), um ácido graxo incomum, que apresenta 18 carbonos na sua estrutura, com uma insaturação cis no carbono 9 e uma hidroxila no carbono 12. Diferentemente da maioria dos óleos vegetais, existem três sítios passíveis de sofrerem modificações: a hidroxila, a insaturação e a carboxila. Dentre estas modificações, a expoxidação da insaturação

"Este artigo é dedicado à Prof. Helena M. C. Ferraz.

*e-mail: mrsnunes@ucs.br no carbono 9 merece destaque especial, uma vez que produzirá um intermediário sintético importante (Esquema 1). Os epóxidos gerados a partir de óleos vegetais podem ser usados diretamente como estabilizantes de PVC, ou então, em uma aplicação mais promissora, serem transformados em polieterpoliois, usados como materiais de partida na produção de poliuretanas. ${ }^{1}$

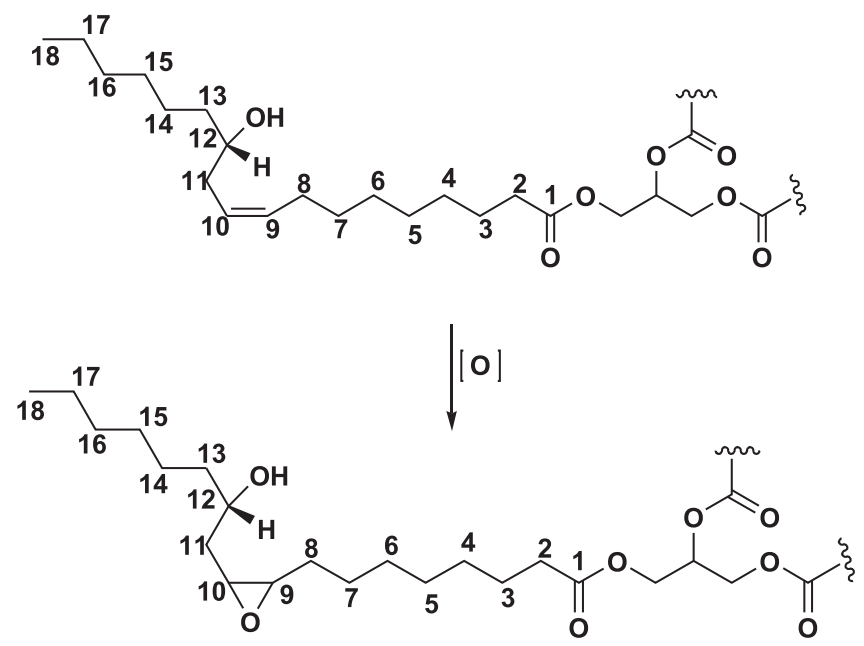

Esquema 1

As reações de epoxidação são usualmente realizadas com ácidos peroxicarboxílicos, como ácido peracético, ácido perfórmico e ácido perbenzóico, porém, estas metodologias apresentam várias desvantagens, como perigo de manuseio em escala industrial, além do alto impacto ambiental devido ao descarte de efluentes do processo. ${ }^{2}$

Mudanças ocorridas no cenário mundial vêm alterando a visão dos pesquisadores e estudantes que atualmente trabalham no sentido de desenvolver processos em consonância com o conceito da chamada "química limpa". Algumas ações podem ser adotadas a fim de diminuir ou eliminar estes problemas, tais como o uso de reagentes alternativos e o uso de catalisadores, o que leva a um 
aumento na eficiência dos processos, facilita a separação de produtos da reação e a reciclagem dos reagentes/catalisadores., ${ }^{3,4}$

Neste contexto, uma área de crescente interesse diz respeito ao uso de catalisadores heterogêneos suportados como alternativa para as mais tradicionais reações orgânicas, bem como o uso de sólidos inorgânicos como meio de se obter algumas importantes transformações químicas. Dentre estes apregoados meios, podem ser destacados os catalisadores heterogêneos, bem como as argilas minerais. Na literatura podem ser encontradas descrições sobre o uso de argilas em reações de transformações de óleos vegetais. ${ }^{5-9}$

A Montmorillonita (K-10) é uma das argilas mais importantes utilizadas em catálise. As propriedades desta argila em aplicações catalíticas são exploradas há décadas e, dentre as suas funções mais importantes podem ser citadas o emprego como agente catalítico ativo (usualmente como ácido sólido), suporte "inerte" ou bifuncional e como filtros para catalisadores sólidos. ${ }^{6,10,11}$

O objetivo deste trabalho foi a investigação sistemática do sistema catalítico $\mathrm{VO}(\mathrm{acac})_{2} / \mathrm{TBHP}$ em reações de epoxidação do óleo de mamona e seus derivados. Neste sistema, o t-butil hidroperóxido (TBHP) é o agente doador de oxigênio na oxidação da dupla ligação presente no substrato insaturado. Adicionalmente, descrevemos alguns resultados preliminares de experimentos realizados em condições reacionais similares, utilizando o catalisador $\mathrm{VO}(\mathrm{acac})_{2}$, suportado em argila K-10.

\section{PARTE EXPERIMENTAL}

Os espectros na região do infravermelho foram registrados em um espectrofotômetro no IV com transformada de Fourier- FT-IR, Shimadzu 8300 (de 500 a $4000 \mathrm{~cm}^{-1}$ ). A análise foi feita em filme entre cristais de $\mathrm{NaCl}$. Os espectros de $\mathrm{RMN}{ }^{1} \mathrm{H}$ e ${ }^{13} \mathrm{C}$ foram obtidos em espectrômetros Varian Inova-300 a 300 MHz. Os deslocamentos foram registrados em unidade $\delta$ (ppm) e o solvente utilizado foi o $\mathrm{CDCl}_{3}$, tendo o tetrametilsilano (TMS) como referência interna. Foi utilizada também a cromatografia gasosa com detector de espectrometria de massas (GC-MSD) para a caracterização dos compostos obtidos, bem como para verificação do grau de pureza dos mesmos. Todos os solventes e reagentes foram purificados conforme métodos usuais, sempre que necessário.

\section{Procedimento geral para a epoxidação do óleo de mamona e derivados}

Em um balão de $25 \mathrm{~mL}$, munido de condensador de refluxo e sob agitação magnética, colocou-se $1,0 \mathrm{~g}$ de óleo ou de seu éster metílico ou etílico derivado e dissolveu-se em $2 \mathrm{~mL}$ de tolueno. A seguir, colocou-se o $\mathrm{VO}(\mathrm{acac})_{2}$ ( $1 \%$ em relação à quantidade de substrato) e, sob agitação intensa, adicionou-se vagarosamente o TBHP em tolueno (proporções utilizadas estão descritas nas Tabelas 2 e 3), observando-se a rápida mudança de coloração. A reação ficou sob agitação a temperatura ambiente ou refluxo do solvente, também conforme descrição feita nas Tabelas 2 e 3. Em seguida, adicionou-se $10 \mathrm{~mL}$ de bissulfito de sódio (30\%) e deixou-se sob agitação por mais 10-15 min. Verificou-se a quantidade de peróxido remanescente com papel indicador de peróxido sendo, a seguir, realizada a extração da fase orgânica com tolueno $(2 \times 10 \mathrm{~mL})$, secagem com $\mathrm{Na}_{2} \mathrm{SO}_{4}$ e evaporação do solvente em evaporador rotatório sob vácuo. $\mathrm{O}$ rendimento do produto em massa, após a concentração, foi de $\sim 900 \mathrm{mg}$. A purificação foi realizada em coluna cromatográfica gradiente em sílica gel (60 a 230 Mesh), utilizando-se uma mistura dos solventes hexano/acetato de etila como eluente. O produto foi obtido numa proporção de $6: 4 /$ hexano:acetato de etila.

\section{Imobilização do reagente $\mathrm{VO}(\mathrm{acac})_{2}$ em argila $\mathrm{K}-10^{12}$}

Em um balão de fundo redondo, sob atmosfera de argônio, foi adicionado o $\mathrm{VO}(\mathrm{acac})_{2}(0,9 \mathrm{mmol}-0,221 \mathrm{~g})$, a argila $\mathrm{K}-10(0,900 \mathrm{~g}$, previamente seca e ativada a $100{ }^{\circ} \mathrm{C}$ ) e $10 \mathrm{~mL}$ de tolueno. A mistura reacional foi deixada durante a noite $(12 \mathrm{~h})$, sendo em seguida o solvente retirado, ainda sob argônio, e secagem final em estufa de $110{ }^{\circ} \mathrm{C} \mathrm{du}-$ rante $8 \mathrm{~h}$. Foram realizadas análises de infravermelho e $\mathrm{MeV}$ a fim de confirmar a imobilização do catalisador na argila K-10.

\section{RESULTADOS E DISCUSSÃO}

Iniciamos os nossos estudos sobre a epoxidação do óleo de mamona utilizando o sistema $\mathrm{VO}(\mathrm{acac})_{2} / \mathrm{TBHP}$, empregando as mesmas condições reacionais descritas por Brasil. ${ }^{13}$ A presença de um grupo hidroxila no carbono 12 da molécula de triricinoleina do óleo de mamona confere maior reatividade à insaturação frente ao emprego de catalisadores de vanádio, conforme dados e propostas mecanísticas encontradas na literatura. Os valores obtidos para conversão da reação, quantidade de epóxido formado e seletividade, utilizando o óleo de mamona e seus derivados, confirmaram esta suposição, uma vez que foram muito melhores quando comparados aos obtidos anteriormente, utilizando o óleo de soja como material de partida.

Segundo Sharpless e colaboradores, a reação de epoxidação de olefinas funcionalizadas, utilizando como catalisador um alcóxido de vanádio, ocorre através de um ciclo catalítico onde o catalisador se complexa com o álcool alílico e com o TBHP, fornecendo o intermediário A que se transformará em $\mathbf{B}$ e $\mathbf{C}$, os intermediários neutros envolvidos na etapa lenta do mecanismo, ou seja, a transferência do oxigênio à olefina (Esquema 2). ${ }^{12}$

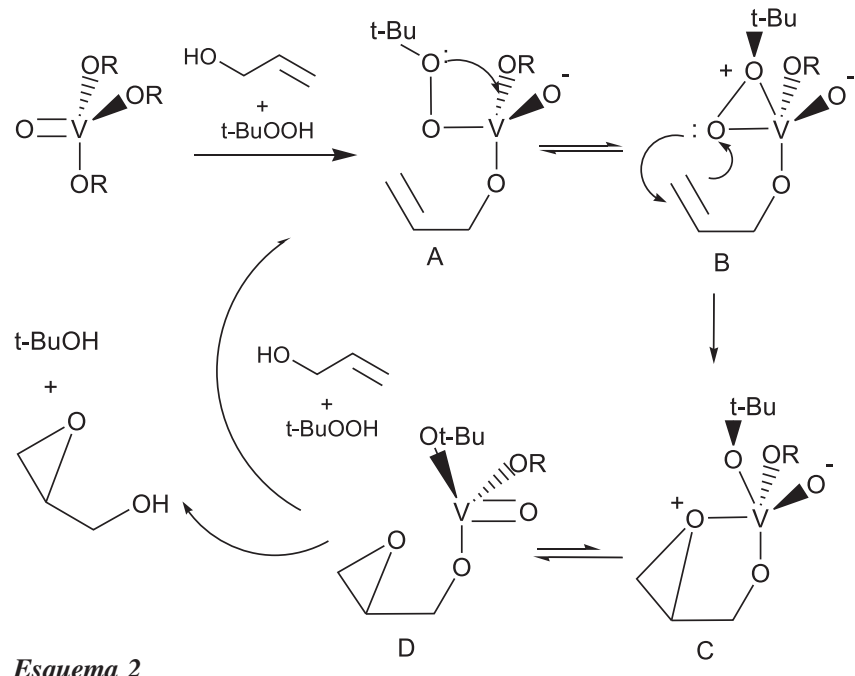

Esquema 2

Na nossa proposta sintética, a molécula alvo possui o grupo hidroxila em uma posição $\beta$ à dupla ligação (C12), tratando-se, portanto, de um álcool homoalílico e não alílico como no mecanismo apresentado no Esquema 2. Entretanto, estudos anteriores demostraram que a epoxidação de alcoóis homoalílicos e bishomoalílicos com o sistema $\mathrm{VO}(\mathrm{acac})_{2} / \mathrm{TBHP}$ levou a produtos com estereosseletividade $\sin$, além de velocidades superiores àquelas obtidas com outros catalisadores, indicando que este sistema catalítico poderia ser efetivo no nosso caso. ${ }^{14}$

Estudos recentes, realizados por Yamamoto, descrevem a epoxidação assimétrica de álcoois homoalílicos, utilizando um catalisador de vanádio associado a diferentes ligantes. Os pesquisadores obtiveram resultados satisfatórios tanto no rendimento da 
reação, quanto na estereosseletividade dos produtos finais, diferentemente dos resultados anteriormente obtidos por Sharpless, também na epoxidação assimétrica de álcoois homoalílicos, utilizando, neste caso, um catalisador de titânio. ${ }^{15}$

Foi realizada a interpretação minuciosa dos sinais característicos de RMN ${ }^{1} \mathrm{H}$ e ${ }^{13} \mathrm{C}$ do óleo de mamona (comercial), utilizando-se esses dados para caracterização dos produtos obtidos e cálculos de conversão, quantidade de epóxido e seletividade das reações realizadas. Através da observação da modificação de sinais específicos nos espectros de $\mathrm{RMN}$ de ${ }^{1} \mathrm{H}$ e ${ }^{13} \mathrm{C}$, foi possível identificar a formação do epóxido. ${ }^{16}$

$\mathrm{Na}$ Tabela 1 podem ser observados os sinais característicos, bem como as principais modificações encontradas no espectro de RMN de ${ }^{1} \mathrm{H}$ do produto epoxidado.

Tabela 1. Comparação entre os valores de deslocamento de RMN de ${ }^{1} \mathrm{H}$ do óleo de mamona e o óleo de mamona epoxidado

\begin{tabular}{|c|c|c|}
\hline \multirow[t]{2}{*}{ Hidrogênios } & \multicolumn{2}{|c|}{ Deslocamento Químico $(\delta, \mathrm{ppm})$} \\
\hline & óleo de mamona & $\begin{array}{c}\text { óleo de mamona } \\
\text { epoxidado }\end{array}$ \\
\hline $\mathrm{CH}_{2} \mathrm{CH}_{3}$ & $0,85-0,89$ & $0,85-0,89$ \\
\hline $\mathrm{CH}_{2} \mathrm{CH}=\mathrm{CHCH}_{2}$ & $2,00-2,07$ e 2,21 & - \\
\hline $\mathrm{HC}(\mathrm{O}) \mathrm{CH}_{2}$ & 2,31 & $\overline{2,31}$ \\
\hline & 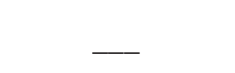 & 2,9 e 3,1 \\
\hline $\mathrm{CH}(\mathrm{OH})$ & $3,58-3,64$ & 3,90 \\
\hline $\mathrm{CH}_{2} \mathrm{OCO}$ & 4,14 e 4,30 & 4,14 e 4,30 \\
\hline$(\mathrm{O}) \stackrel{2}{\mathrm{C}} \mathrm{H}_{2} \mathrm{CH}(\mathrm{O}) \mathrm{CH}_{2}(\mathrm{O})$ & $5,26-5,31$ & $5,26-5,31$ \\
\hline $\mathrm{CH}_{2} \mathrm{C} \boldsymbol{H}=\mathrm{CHCH}_{2}$ & $5,34-5,60$ & - \\
\hline
\end{tabular}

Nos espectros de RMN de ${ }^{13} \mathrm{C}$, as principais modificações observadas referem-se ao desaparecimento dos carbonos olefínicos em 125,1 e 130,6 ppm, e ao aparecimento dos carbonos nas regiões de 55,4 e 56,3 ppm, característicos do anel oxirânico. ${ }^{17}$

Após a purificação dos produtos de reação através de coluna cromatográfica de sílica gel, foi realizada a derivatização da amostra e posterior análise por CG/EM. O principal componente encontrado na amostra analisada foi o metil-cis-9,10-epoxiesterato (82\%), confirmando a seletividade atribuída às reações realizadas empregando-se catalisadores de vanádio e consistente com o mecanismo proposto, onde a transferência do átomo de oxigênio ocorre através de um complexo intramolecular. ${ }^{12-15}$

Os valores de conversão, grau de epoxidação e seletividade da reação foram calculados com base nos dados de $\mathrm{RMN}{ }^{1} \mathrm{H}$ do óleo de partida e do produto formado (Tabela 2).

Tabela 2. Reações de epoxidação do óleo de mamona utilizando o sistema $\mathrm{VO}(\mathrm{acac})_{2} / \mathrm{TBHP}^{\mathrm{a}}$

\begin{tabular}{lcccc}
\hline Reação & $\begin{array}{c}\text { Tempo } \\
(\mathrm{h})\end{array}$ & $\begin{array}{c}\text { Conversão } \\
(\mathrm{C}, \%)\end{array}$ & $\begin{array}{c}\text { Epóxido } \\
(\mathrm{E}, \%)\end{array}$ & $\begin{array}{c}\text { Seletividade } \\
(\mathrm{S}, \%)\end{array}$ \\
\hline 1 & 5 & 78 & 61 & 78 \\
$2^{\mathrm{b}}$ & 5 & 77 & $62(51)^{\mathrm{c}}$ & 80 \\
3 & 7 & 92 & 65 & 70 \\
$4^{\mathrm{d}}$ & 3 & 88 & 73 & 82 \\
$5^{\mathrm{e}}$ & 3 & 94 & 68 & 73 \\
$6^{\mathrm{f}}$ & 22 & 96 & 71 & 74 \\
\hline
\end{tabular}

a) $1 \mathrm{~g}$ de óleo (3,6 mmol), $\mathrm{VO}(\mathrm{acac})_{2}(1 \%)$, TBHP (3,6 mmol), 2 $\mathrm{mL}$ de tolueno; refluxo; b) reação realizada com $3 \mathrm{~g}$ de óleo; c) determinado através do índice de oxigênio oxirânico; d) excesso de $20 \%$ de TBHP; e) excesso de $40 \%$ de TBHP; f) condição (a), porém realizada à temperatura ambiente.
Nas reações 1 e $\mathbf{2}$, onde somente foram realizadas variações na quantidade de amostra utilizada (1 para $3 \mathrm{~g}$ ), não ocorrem alterações significativas nos valores de $\mathbf{C}, \mathbf{E}$ e $\mathbf{S}$ (conversão, quantidade de epóxido e seletividade, respectivamente). A divergência dos rendimentos da quantidade de epóxido da reação $\mathbf{6}$, onde o valor percentual determinado através do oxigênio oxirânico foi menor, pode ser atribuída ao fato de não ter sido realizada a purificação prévia da amostra. Uma vez que foi realizada somente uma filtração para a retirada do catalisador do meio reacional, a amostra poderia conter resíduos de vanádio, podendo interferir na determinação da quantidade de epóxido.

Na reação 3, onde o tempo reacional foi alterado de 5 para $7 \mathrm{~h}$, pode ser observada uma grande variação no percentual de conversão da reação, porém com uma diminuição significativa na seletividade $(\mathbf{S})$.

Finalmente, foi realizado um experimento utilizando um excesso do agente oxidante (20\%) nas condições de refluxo do solvente, sendo observado um alto percentual de conversão da reação (88\%), e rendimentos satisfatórios para formação do epóxido (73\%) e seletividade ( $82 \%$ ), o que nos levou a acreditar que um aumento na quantidade de agente epoxidante levaria a uma melhora nos resultados. Em razão disto, realizou-se um novo experimento, desta vez utilizando $40 \%$ de excesso de TBHP. Entretanto, conforme o resultado apresentado na Tabela 1 (reação 5), houve um aumento no valor de conversão $(94 \%)$, porém com resultados menores para epóxido e seletividade (68 e 73\%, respectivamente).

Foi realizada ainda, uma reação a temperatura ambiente (reação 6), onde, a exemplo de reações anteriores, obteve-se um alto percentual de conversão e valores menores de seletividade (S) e epóxido $(\mathbf{E})$.

A ocorrência da menor seletividade das reações $\mathbf{3}, \mathbf{5}$ e $\mathbf{6}$ pode ser atribuída à formação de produtos secundários provenientes da abertura do epóxido durante o curso da reação. A confirmação de polióis como compostos secundários da reação de epoxidação foi realizada através das análises de $\mathrm{RMN}$ de ${ }^{1} \mathrm{H}$, onde se observou a presença de sinais com valores de deslocamento entre 3,0 e 4,5 ppm, característicos destes subprodutos.

A fim de comprovar a generalidade deste método, e verificar se alterações significativas na estrutura dos óleos levariam a melhores resultados em relação à seletividade e formação do epóxido, foram realizados experimentos utilizando derivados do óleo de mamona, os ésteres metílico e etílico. Neste caso, apesar dos valores consideravelmente altos de conversão do substrato, os demais resultados obtidos foram similares aos do óleo de mamona, conforme pode ser observado na Tabela 3 .

Tabela 3. Reações de epoxidação dos ésteres do óleo de mamona utilizando o sistema $\mathrm{VO}(\mathrm{acac})_{2} / \mathrm{TBHP}$

\begin{tabular}{lcccc}
\hline Substrato & $\begin{array}{c}\text { Tempo } \\
(\mathrm{h})\end{array}$ & $\begin{array}{c}\text { Conversão } \\
(\%, \mathrm{C})\end{array}$ & $\begin{array}{c}\text { Epóxido } \\
(\%, \mathrm{E})\end{array}$ & $\begin{array}{c}\text { Seletividade } \\
(\%, \mathrm{~S})\end{array}$ \\
\hline Éster Metílico $^{\mathrm{a}}$ & 3 & 94 & 76 & 81 \\
Éster Metílico $^{\mathrm{b}}$ & 3 & 95 & 85 & 89 \\
Éster Etílico $^{\mathrm{c}}$ & 3 & 100 & 73 & 73 \\
\hline
\end{tabular}

a) $1 \mathrm{~g}$ do éster metílico (1,14 mmol), $\mathrm{VO}(\mathrm{acac})_{2}$ (1\% ), TBHP (1,37 mmol, 20\% de excesso), $2 \mathrm{~mL}$ de tolueno, refluxo; b) condições (a), porém $40 \%$ de excesso de TBHP; c) $1 \mathrm{~g}$ do éster etílico (1,09 mmol), $\mathrm{VO}(\mathrm{acac})_{2}(1 \%)$, TBHP (1,30 mmol, $20 \%$ de excesso), 2 $\mathrm{mL}$ de tolueno, refluxo.

\section{Utilização da argila K-10 na epoxidação do óleo de mamona}

Foram realizados testes preliminares com a argila K-10 e o óleo de mamona visando investigar o potencial desta argila para 
reações de epoxidação. Nos experimentos realizados para a epoxidação do óleo de mamona, empregando a mesma metodologia descrita anteriormente ( $\mathrm{VO}(\mathrm{acac})_{2} / \mathrm{TBHP}$, tolueno/5 h de refluxo) com a simples adição da argila ao meio reacional, foi observada, além da conversão quantitativa do material de partida e formação do epóxido (RMN de ${ }^{1} \mathrm{H}$, sinais em 2,9 e 3,1 ppm), uma grande proporção de produtos da provável abertura do anel (sinais em 3,7 e 4,1 ppm). Entretanto, quando a reação foi conduzida a temperatura ambiente ( $24 \mathrm{~h}$ de agitação), os seguintes resultados foram obtidos: $83,3 \%$ de conversão, $46,8 \%$ de epóxido e $56,2 \%$ de seletividade. Observou-se também que houve a degradação de parte do produto da reação, confirmado pela presença dos mesmos sinais referentes à abertura do epóxido. Estes resultados prévios levam a acreditar que a K-10 promove um aumento na velocidade de reação, mas exige condições mais brandas para que esta transformação aconteça (aquecimento leve ou menor tempo reacional de refluxo).

Apesar das análises realizadas (IV e MeV) não fornecerem resultados conclusivos em relação ao percentual obtido de imobilização do reagente de vanádio na argila, foram conduzidos alguns experimentos com o catalisador $\mathrm{VO}(\text { acac })_{2} / \mathrm{K}-10$, preparado conforme descrito na parte experimental. Foram encontrados valores de conversão de $100 \%$, formação do epóxido de $67 \%$ e seletividade de $67 \%$, para a reação realizada em 2 h de refluxo de tolueno e 25 $\mathrm{mg}$ do catalisador de vanádio imobilizado. Estes resultados foram melhores que os obtidos anteriormente, mas ainda insatisfatórios quando comparados aos das reações utilizando-se somente o sistema $\mathrm{VO}(\mathrm{acac})_{2} / \mathrm{TBHP}$. Foram realizadas reações complementares variando-se o tempo reacional ( 3 e $5 \mathrm{~h}$ ) e a quantidade de reagente imobilizado (50 mg), sem grandes alterações nos resultados já descritos.

\section{CONCLUSÃO}

Os resultados obtidos neste trabalho demonstram o potencial do sistema catalítico $\mathrm{VO}(\mathrm{acac})_{2} / \mathrm{TBHP}$ na epoxidação do óleo de mamona, bem como seus ésteres derivados. Estas reações foram realizadas em condições brandas e sob catálise heterogênea, consonantes com o desenvolvimento de tecnologias voltadas à trans- formação de óleos vegetais dentro dos preceitos da química limpa: eficiência de energia pela diminuição de etapas de reação e reações mais seletivas, emprego de catalisador de natureza sólida em substituição a reações estequiométricas, geração de produtos biodegradáveis, diminuição na quantidade de reagentes para a prevenção da poluição. Encontra-se sob investigação a eficiência do sistema no qual o $\mathrm{VO}$ (acac), foi imobilizado em argila K10, uma vez que os resultados preliminares não foram conclusivos.

\section{REFERÊNCIAS}

1. Schneider, R. C. S.; Tese de Doutorado, Universidade Federal do Rio Grande do Sul, Brasil, 2003; Metzer, J. O.; Bornscheuer, U; Appl. Microbiol. Biotechnol. 2006, 71, 13; Lligadas, G.; Ronda, J. C.; Galià, M.; Biermann, U.; Metzger, J. O.; J. Polym. Sci., Part A: Polym. Chem. 2006, 44, 634 .

2. Von Holleben, M. L. A.; Schuch, C. M.; Quim. Nova 1997, 20, 58; Gerbase, A. E.; Brasil, M. C.; Gregório, J. R.; Mendes, A. N. F.; Von Holleben, L. A.; Martinelli, M.; Grasas y Aceites 2002, 53, 175.

3. Lenardão, E. J.; Quim. Nova 2003, 26, 123.

4. Clark, J. H.; Wilson, K.; Chem. Commun. 1998, 19, 2135.

5. Price, P. M., Clark, J. H.; Macquarrie, D. J.; Dalton Trans. 2000, 2, 101

6. Varma, R. S.; Tetrahedron 2002, 58, 1235.

7. Rodrigo, M. T.; Mendioroz, S.; Daza, L.; Appl. Catal, A 1992, 88, 101.

8. Taqui Khan, M. M.; Samad, S. A.; Siddiqui, M. R. H.; J. Mol. Catal. A: Chem. 1989, 50, 97.

9. Poels, E. K.; Bliek, A.; Leeuw, B.; Bogert, M.; Koster, R. M.; J. Mol. Catal. A: Chem. 1998, 134, 159.

10. Palombi, L.; Bonadies, F.; Scettri, A.; J. Mol. Catal. A: Chem. 1999, 140, 47.

11. Gournis, D.; Louloudi, M.; Karakassides, M. A.; Kolokytha, C.; Mitopoulou, K.; Hadjiliadis, N.; Mater. Sci. Eng. C 2002, 22, 113.

12. Sharpless, K. B.; Verhoeven, T. R.; Aldrichimica Acta 1979, 12 , 63;Sharpless, K. B.; Michaelson, R. C.; J. Am. Chem. Soc. 1973, 95, 6136; Choudary, B. M.; Valli, V. L. K.; Prasad, D.; Chem. Commun. 1990, 9, 721.

13. Brasil, M. C.; Dissertação de Mestrado, Universidade Federal do Rio Grande do Sul, Brasil, 2000.

14. Mihelich, E. D.; Daniels, K.; Eickhoff, D. J.; J. Am. Chem. Soc. 1981, 103, 7690; Hoveydo, A. H.; Evans, D. A.; Fu, G. C.; Chem. Rev. 1993, 93, 1307; Lattanzi, A.; Leadbeater, N. E.; Org. Lett. 2002, 4, 1519.

15. Sharpless, K. B.; Rossiter, B. R.; J. Org. Chem. 1984, 49, 3707; Yamamoto, H.; Zhang, W.; J. Am. Chem. Soc. 2007, 129, 286.

16. Crivello, J. V.; Chakrapani, S.; J. Macromol. Sci., Part A: Pure Appl. Chem. 1998, $A 35,1$.

17. Lie Ken Jie, M. S. F.; Lam, C. K.; Ultrason. Sonochem. 1995, 2, 11. 